\title{
Search for Cosmic-Ray Antideuterons with BESS-Polar II
}

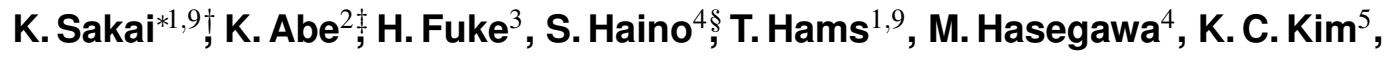 \\ M. H. Lee ${ }^{5}$, Y. Makida ${ }^{4}$, J. W. Mitchell ${ }^{1}$, J. Nishimura ${ }^{6}$, M. Nozaki ${ }^{4}$, R. Orito ${ }^{2}$, \\ J. F. Ormes ${ }^{7}$, N. Picot-Clemente ${ }^{5}$, M. Sasaki ${ }^{1,9}$, E. S. Seo ${ }^{5}$, R. E. Streitmatter ${ }^{1}$, \\ J. Suzuki ${ }^{4}$, K. Tanaka ${ }^{4}$, N. Thakur ${ }^{1}$, A. Yamamoto ${ }^{4}$, T. Yoshida ${ }^{3}$ and K. Yoshimura ${ }^{8}$ \\ ${ }^{1}$ NASA-Goddard Space Flight Center (NASA-GSFC), Greenbelt,MD 20771, USA \\ ${ }^{2}$ Kobe University, Kobe, Hyogo 657-8501, Japan \\ ${ }^{3}$ Institute of Space and Astronautical Science, Japan Aerospace Exploration Agency \\ (ISAS/JAXA), Sagamihara, Kanagawa 252-5210, Japan \\ ${ }^{4}$ High Energy Accelerator Research Organization (KEK), Tsukuba, Ibaraki 305-0801, Japan \\ ${ }^{5}$ IPST, University of Maryland, College Park, MD 20742, USA \\ 6 The University of Tokyo, Bunkyo, Tokyo 113-0033, Japan \\ ${ }^{7}$ University of Denver, Denver, CO 80208, USA \\ ${ }^{8}$ Okayama University, Okayama, Okayama 700-0082, Japan \\ ${ }^{9}$ Center for Research and Exploration in Space Science and Technology (CRESST)
}

\begin{abstract}
High-precision measurements of the cosmic-ray antiproton spectrum and sensitive search for cosmological antihelium have been published using the data from BESS-Polar II (Balloon-borne Experiment with a Superconducting Spectrometer) flight in 2007/2008 for a core study of the early Universe using elementary particle measurements. The most sensitive antideuteron search reported used the data obtained by BESS97, BESS98, BESS99, and BESS00, which include the solar minimum period in 1997 . We performed a search for antideuterons with unprecedented sensitivity using BESS-Polar II data that is more than ten times the statistics of BESS97 in near solar minimum conditions. The search for antideuteron probes possible exotic sources, such as dark-matter candidates.
\end{abstract}

36th International Cosmic Ray Conference -ICRC2019-

July 24th - August 1st, 2019

Madison, WI, U.S.A.

\footnotetext{
* Speaker.

†E-mail: kenichi.sakai@nasa.gov

$\ddagger$ Present address: Kamioka Observatory, ICRR, The University of Tokyo, Hida, Gifu 506-1205, Japan.

${ }^{\S}$ Present address: Institute of Physics, Academia Sinica, Nankang, Taipei 11529, Taiwan.

๑Present address: Tokushima University, Tokushima, Tokushima 770-8502, Japan.
} 


\section{Introduction}

The precise measurement of the cosmic-ray antiproton spectrum $(\bar{p}$ 's) is crucially important to the investigation of elementary particle phenomena in the early Universe. Most of the observed cosmic-ray antiprotons are well understood as secondary products of collisions between primary cosmic-rays and the interstellar medium. The energy spectrum of such "secondary" antiprotons peaks near $2 \mathrm{GeV}$, and decreases sharply below and above the peak, due to the kinematics of antiproton production and to the local interstellar (LIS) proton spectrum.

Cosmic-ray antideuterons ( $\bar{d}$ 's) are in a different situation. Thanks to their heavier mass, the probability of production in cosmic-ray interactions is much smaller, especially at low energies, because of the very low production cross-section and strict kinematical requirements. So one single $\bar{d}$ event would be a direct evidence of novel primary origins. However, the expected flux is extremely small. The more numerous $\bar{p}$ 's are a severe background in identifying $\bar{d}$ 's. An experiment with large exposure factor and good particle identification devices is necessary to investigate $\bar{d}$ 's. As such, few searches have been carried out so far and no $\bar{d}$ candidate was observed yet. The best limit was reported by the BESS experiment obtained during last solar minimum period [1].

\section{BESS Program}

The BESS instrument $[2,3]$ was developed as a high-resolution magnetic-rigidity spectrometer for cosmic-ray antiparticles and precise measurements of the absolute fluxes of various cosmic-ray components. The original BESS experiment performed 9 flights over northern Canada during the period of 1993 through 2002 with continuous improvement in the instrument. The BESS-

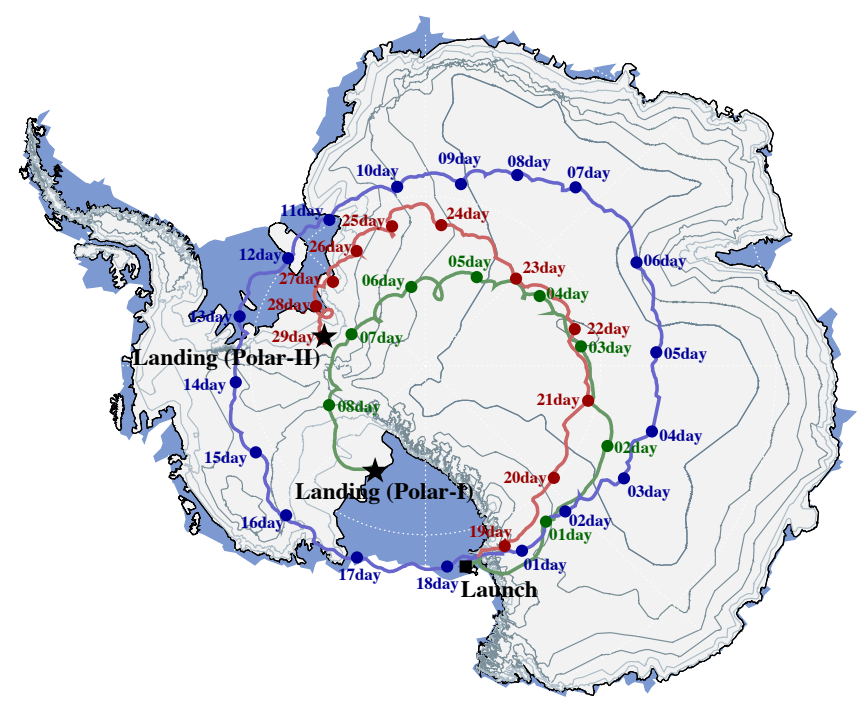

Figure 1: Flight trajectory of the 2007 BESS-Polar II over Antarctica from Williams Field (first orbit blue, second orbit red) with 2004 BESS-Polar I flight (green).

[Launch]S77-51,E166-40, 06:27(McM) 12/23 2007

[Recovery]S83-51,W073-04, 09:02(UTC) 1/21 2008 


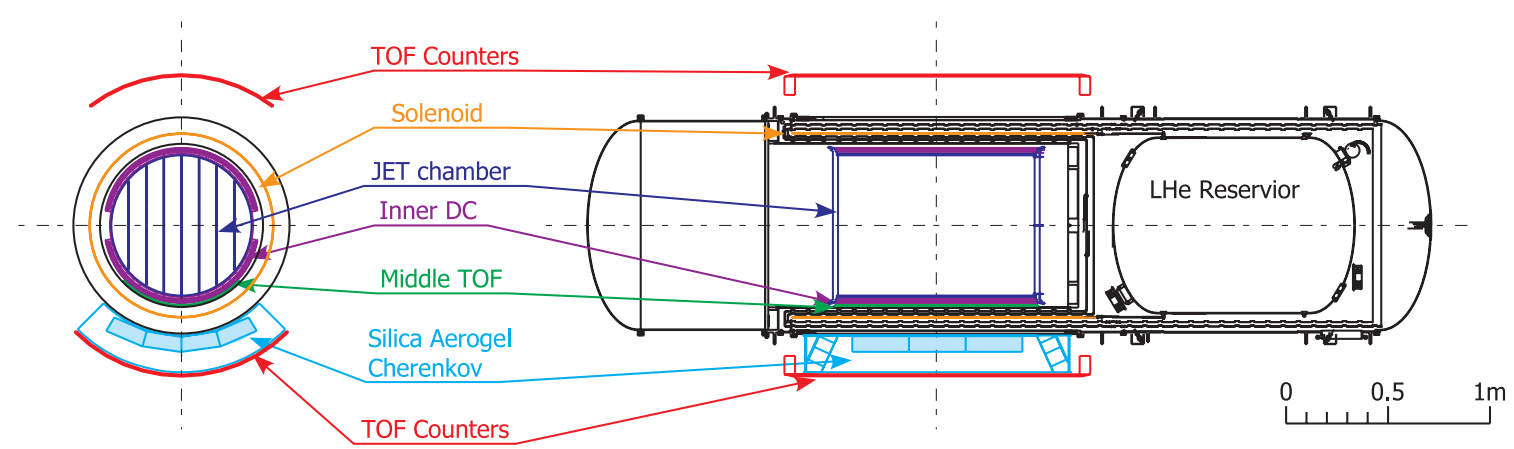

Figure 2: Cross sectional view of BESS-Polar II spectrometer

Polar project was proposed as an advanced BESS program using long duration balloon (LDB) flights over Antarctica (around the south pole) to provide high-statistics, low-energy cosmic-ray measurements $[4,5,6]$. The first scientific flight of the BESS-Polar instrument was launched near McMurdo Station, on December 13th, 2004 (UTC). The flight duration was over 8.5 days and more than $9 \times 10^{8}$ cosmic-ray events were recorded [7]. Incorporating considerable improvements in instrument and payload systems compared to BESS-Polar I, the BESS-Polar II instrument was launched on December 23, 2007, from Williams Field near the US McMurdo Station in Antarctica and circulated around the South Pole for 24.5 days of observation with the magnet energized. The float altitude was $34 \mathrm{~km}$ to $38 \mathrm{~km}$ (residual air of $5.8 \mathrm{~g} / \mathrm{cm}^{2}$ on average), and the cutoff rigidity was below $0.5 \mathrm{GV}$. BESS-Polar II accumulated $4.7 \times 10^{9}$ events with no inflight event selection as 13.6 terabytes of data (Fig. 1).

The BESS-Polar II program [8] has produced three papers giving precise measurements of antiprotons [9], a sensitive antihelium search [10] and the absolute spectra for cosmic-ray protons and helium nuclei [11]. The antiproton spectrum measured by BESS-Polar II shows good consistency with secondary antiproton calculations and no evidence of primary antiprotons originating from the evaporation of primordial black holes. And antihelium work has set a new limit in the ratio of possible antihelium to measured helium of $6.9 \times 10^{-8}$ at $95 \%$ confidence, the lowest limit to date. Proton and helium spectra observed from PAMELA [12, 13], AMS-02 [14, 15] and BESS-Polar agree within one $\sigma_{E}$ at high-energies.

\section{BESS-Polar Instrument}

In the BESS-Polar instruments shown in Fig. 2, a uniform field of $0.8 \mathrm{~T}$ is produced by a thin superconducting solenoid, and the field region is filled with drift-chamber tracking detectors. Tracking is performed by fitting up to 52 hit points with a characteristic resolution of $\sim 140 \mu \mathrm{m}$ in the bending plane, resulting in a magnetic-rigidity $(\equiv P c / Z e)$ resolution of $0.4 \%$ at $1 \mathrm{GV}$ and a maximum detectable rigidity (MDR) of $240 \mathrm{GV}$. Upper and lower scintillator hodoscopes provide time-of-flight (TOF) and $d E / d x$ measurements and the event trigger. For antiproton measurements, the acceptance of BESS-Polar is $0.23 \mathrm{~m}^{2} \mathrm{sr}$ and for proton and helium measurements the acceptance is $0.18 \mathrm{~m}^{2} \mathrm{sr}$. The timing resolution of the TOF system is $120 \mathrm{ps}$, giving a $\beta^{-1}$ resolution of $2.5 \%$. The instrument also incorporates a threshold-type Cherenkov counter using a silica aerogel 

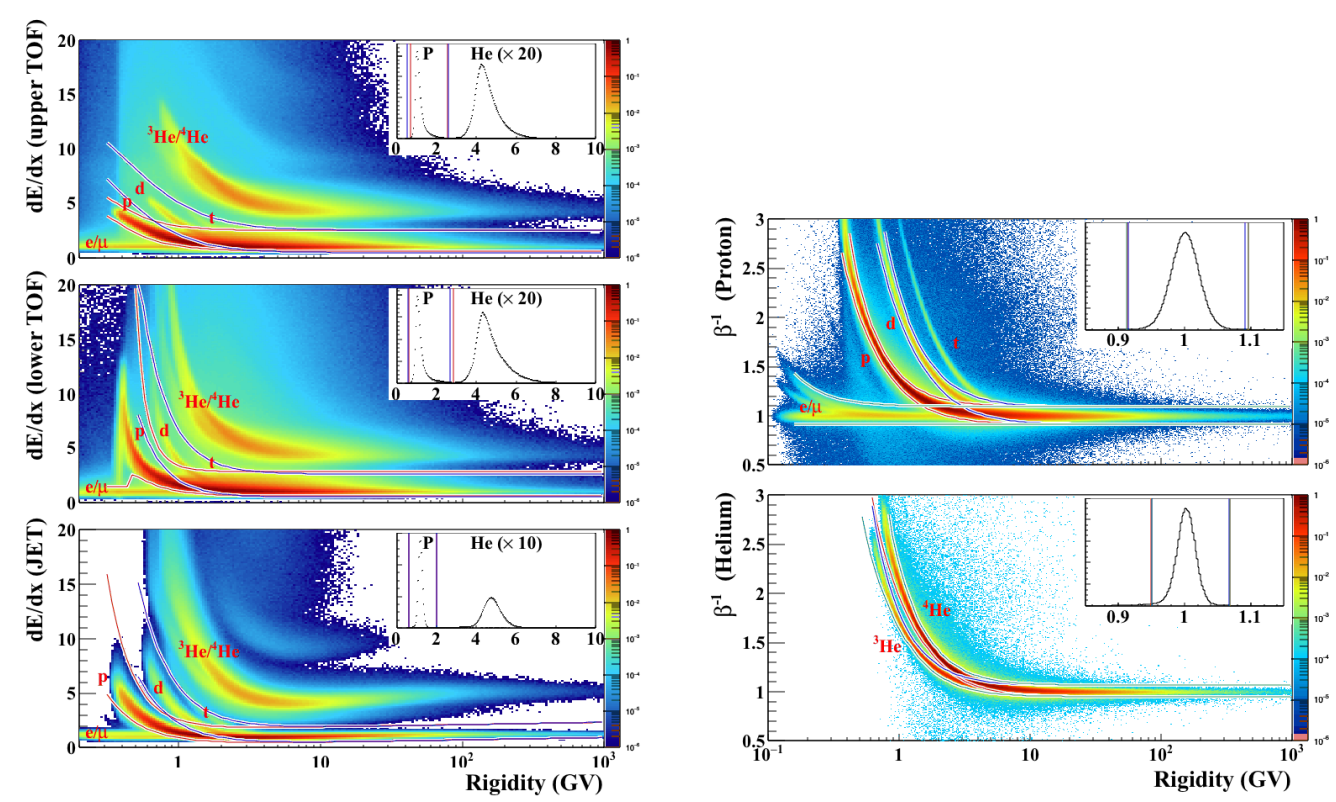

Figure 3: Proton and deuteron bands in $d E / d x$ Figure 4: BESS-Polar II proton, deuteron (top) vs $R$ (top: UTOF, middle: LTOF, bottom: JET) and helium (bottom) bands in $\beta^{-1}$ vs $R$ after the from BESS-Polar II. The superimposed lines selections shown in Fig. 3. The superimposed show the selection criteria for $\mathrm{Z}=1$ and $\mathrm{Z}=2$ par- lines show the selection criteria for protons and ticles. The inset graphs show the results of those helium. The inset graphs show the results of selections above $30 \mathrm{GV}$. those selections above $30 \mathrm{GV}$.

radiator with index $n=1.03$ (ACC) that can reject $e^{-}$and $\mu^{-}$backgrounds by a factor of 12000 and distinguish $\bar{p}$ 's from such backgrounds up to $3.5 \mathrm{GeV}$.

\section{Data analysis}

In the first stage of data analysis, we selected events with a single track fully contained inside the fiducial volume defined by the central four columns out of eight columns in the JET chamber. This definition of the fiducial volume reduced the effective geometrical acceptance down to $\sim \frac{1}{3}$ of the full acceptance, but it ensured the longest track fitting and thus the highest resolution in the rigidity measurement. A single-track event was defined as an event which has only one isolated track and one or two hit counters in each layer of the TOF hodoscopes. The single-track selection eliminated rare interacting events. To estimate the efficiency of the single-track selection, Monte Carlo simulations with GEANT4 were performed. At this point, the same selection criteria for positive and negative curvature events are applied under the assumption that non-interactive $\bar{d}(\bar{p})$ behave like $d(p)$ except for their deflection thanks to the cylindrical symmetry of the BESS-Polar spectrometer. We can also estimate various efficiencies for $\bar{d}$ 's by using positive curvature events (d). The selection criteria of $\bar{d}$ are basically identical to what were applied in $\bar{p}$ analysis. To prevent $\bar{p}$ background contamination into $\bar{d}$, improved tracking quality was required by eliminating noisy IDC hit events, and selecting events that pass through center region of JET. 
$d$ and $\bar{d}$ candidates are then selected by using combination of $d E / d x$ and $\beta$ information. We first apply band selections according to $d E / d x$ for upper and lower TOF and JET chamber. Figure 3 shows $d E / d x$ vs rigidity plot for each detector. We require that $\bar{d}$ 's as well as $d$ 's must have the $d E / d x$ inside the band shown in Fig. 3. Figure 4 shows scatter plot of $\beta^{-1}$ vs rigidity after the selections shown in Fig. 3.

\section{Antideuteron identification}

Figure 5 shows surviving single-charge events in $\beta^{-1}$ vs rigidity plot after $d E / d x$ and ACC cut. Higher ACC threshold was employed to prevent background contamination of relativistic e/ $\mu / \pi$ samples as well as $\bar{p}$ 's. The rejection power is 43000 , more than three times of that in the $\bar{p}$ analysis. Blue shaded band denotes signal region for $\bar{d}$ excluding $3.5 \sigma$ region from $\bar{p}$ center to prevent $\bar{p}$ contamination. While total number of background is calculated to be 0.3 events, we found no $\bar{d}$ candidates.

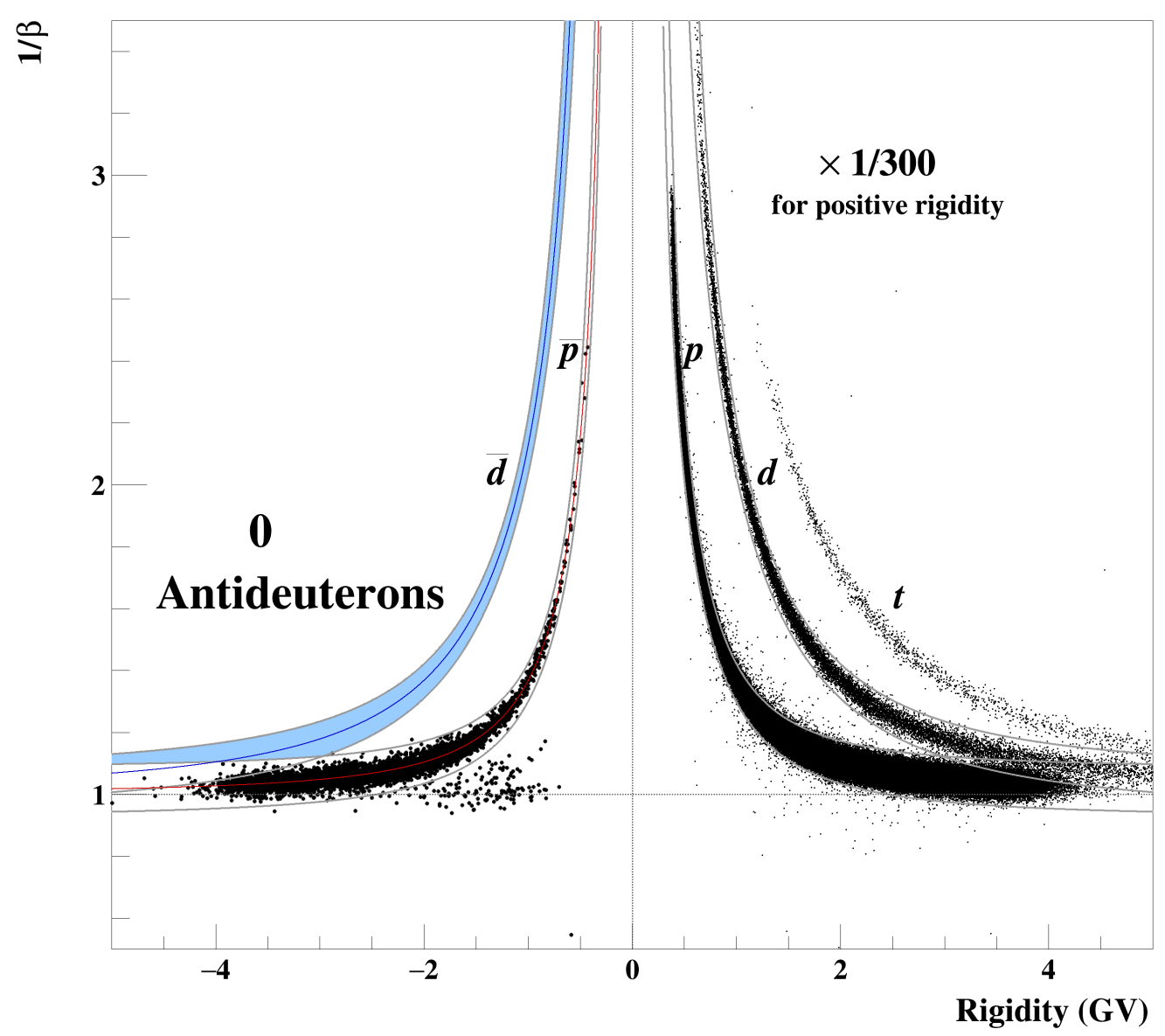

Figure 5: Surviving single-charge events in $\beta^{-1}$ vs rigidity plot after $d E / d x$ and ACC cut in BESS-Polar II data 


\section{Conclusion}

Since no $\bar{d}$ candidate was found, we calculated the resultant 95\% C.L. upper limit on the deuteron flux. It will be reported in the conference.

\section{Acknowledgements}

The BESS-Polar program is a Japan-United States collaboration, supported in Japan by the Grant-in-Aid 'KAKENHI' for Specially Promoted and Basic Researches, MEXT-JSPS, and in the U.S. by NASA. Balloon flight operations were carried out by the NASA Columbia Scientific Balloon Facility and the National Science Foundation United States Antarctic Program. We would like to express our sincere thanks for their continuous professional support.

\section{References}

[1] H. Fuke et al., Search for cosmic-ray antideuterons, Phys. Rev. Lett. 95 (Aug, 2005) 081101.

[2] BESS Collaboration, Y. Ajima et al., A superconducting solenoidal spectrometer for a balloon- borne experiment, Nucl. Instrum. Meth. A443 (2000) 71.

[3] S. Haino et al., Progress of the BESS superconducting spectrometer, Nucl. Instrum. Meth. A518 (2004) 167.

[4] A. Yamamoto et al., BESS and its future prospect for polar long duration flights, Adv. Space Res. 30 (2002) 1253.

[5] J. W. Mitchell et al., The BESS program, Nucl. Phys. (Proc. Suppl.) 134 (2004) 31.

[6] T. Yoshida et al., BESS-polar experiment, Adv. Space Res. 33 (2004) 1755.

[7] K. Abe et al., Measurement of cosmic-ray low-energy antiproton spectrum with the first BESS-Polar Antarctic flight, Phys. Lett. B670 (2008) 103.

[8] K. Abe et al., The results from bess-polar experiment, Advances in Space Research (In Press) (2016).

[9] K. Abe et al., Measurement of the cosmic-ray antiproton spectrum at solar minimum with a long-duration balloon flight over antarctica, Phys. Rev. Lett. 108 (Jan, 2012) 051102.

[10] K. Abe et al., Search for antihelium with the bess-polar spectrometer, Phys. Rev. Lett. 108 (Mar, 2012) 131301.

[11] K. Abe et al., Measurements of cosmic-ray proton and helium spectra from the bess-polar long-duration balloon flights over antarctica, Astrophys. J. 822 (2016), no. 265 (16pp).

[12] O. Adriani et al., Pamela measurements of cosmic-ray proton and helium spectra, Science 332 (2011), no. $602569-72$.

[13] O. Adriani et al., The pamela mission: Heralding a new era in precision cosmic ray physics, Phys. Rep. 544 (2014), no. 4 323-370.

[14] M. Aguilar et al., Precision measurement of the proton flux in primary cosmic rays from rigidity $1 \mathrm{gv}$ to 1.8 tv with the alpha magnetic spectrometer on the international space station, Phys. Rev. Lett. 114 (Apr, 2015) 171103.

[15] M. Aguilar et al., Precision measurement of the helium flux in primary cosmic rays of rigidities $1.9 \mathrm{gv}$ to 3 tv with the alpha magnetic spectrometer on the international space station, Phys. Rev. Lett. 115 (Nov, 2015) 211101 (9pp). 